\title{
Caspase-Cleaved Transactivation Response DNA-Binding Protein 43 in Parkinson's Disease and Dementia with Lewy Bodies
}

\author{
Polina Kokoulina Troy T. Rohn \\ Department of Biology, Boise State University, Boise, Idaho, USA
}

\section{Key Words}

Transactivation response DNA-binding protein 43

proteinopathies $\cdot$ Parkinson's disease $\cdot$ Dementia with Lewy bodies $\cdot \alpha$-Synucleinopathies $\cdot$ Hirano bodies .

$\alpha$-Synuclein · Caspases

\begin{abstract}
Background: Transactivation response DNA-binding protein 43 (TDP-43) proteinopathies are classified based upon the extent of modified TDP-43 and include a growing number of neurodegenerative diseases such as amyotrophic lateral sclerosis, frontotemporal lobar degeneration with ubiquitin-immunoreactive, tau-negative inclusions and frontotemporal lobar degeneration with motor neuron disease. Objective: The purpose of the study was to examine whether proteolytic modifications of TDP-43 are a relevant finding in Parkinson's disease (PD) and dementia with Lewy bodies (DLB). Methods: A novel site-directed caspase cleavage antibody, termed TDP caspase cleavage product antibody (TDPccp), was utilized based upon a known caspase 3 cleavage consensus site within TDP-43 at position 219. Results: Application of this antibody to postmortem brain sections from $P D$ and DLB patients revealed the presence of caspasecleaved TDP-43 in Lewy bodies and Hirano bodies in all cases examined. Colocalization of TDPccp with an antibody to
\end{abstract}

$\alpha$-synuclein ( $\alpha$-Syn), which served as a general marker for Lewy bodies, was evident within the substantia nigra in both $\alpha$-synucleinopathies. Interestingly, the TDPccp antibody detected a greater number of Lewy bodies in PD and DLB compared to the $\alpha$-Syn antibody. In addition, a semiquantitative analysis in both diseases confirmed this finding by indicating that the percentage of caspase-cleaved TDP-43 single-labeled Lewy bodies was approximately twice that of $\alpha$-Syn labeling (in DLB 13.4 vs. 5.5\%, while in PD 34.6 vs. 17.6\%). Conclusion: Collectively, these data have identified caspasecleaved TDP-43 as a primary component of Lewy and Hirano bodies in PD and DLB, and suggest that the TDPccp antibody is an effective marker for the detection of Lewy bodies in these neurodegenerative diseases.

Copyright $\odot 2010$ S. Karger AG, Basel

\section{Introduction}

Transactivation response DNA-binding protein 43 (TDP-43) is a highly conserved 414-amino-acid protein with an apparent molecular weight of approximately 43 $\mathrm{kDa}$. It is ubiquitously expressed and appears to play a role in regulating RNA transcription and alternative splicing [1]. Findings from a recent study have also linked TDP-43 function to cytoskeletal stability and axonal

\section{KARGER}

() 2010 S. Karger AG, Basel

Fax +4161306 1234

E-Mail karger@karger.ch

www.karger.com
Accessible online at:

www.karger.com/ndd
Troy T. Rohn, $\mathrm{PhD}$

Department of Biology, Boise State University

Science/Nursing Building, Room 228

Boise, ID 83725 (USA)

Tel. +1 208426 2396, Fax +1 208426 4267, E-Mail trohn@boisestate.edu 
transport by showing that TDP-43 regulates human neurofilament RNA stability [2].

TDP-43 has been identified as a major component of ubiquitinated tau-negative inclusions in sporadic and familial frontotemporal lobar degeneration (FTLD-U) and amyotrophic lateral sclerosis (ALS) [3]. A conspicuous finding in these studies was the presence of 25- and 35$\mathrm{kDa}$ truncated fragments of TDP-43 in brain extracts from affected individuals, which were not present in control subjects [3]. For this common pathology, these diseases were grouped together as a new entity of neurodegenerative disorders, classified as TDP-43 proteinopathies [4]. In addition, it has been recently reported that TDP-43-positive inclusions occur in other neurodegenerative disorders including brains of patients with argyrophilic grain disease, Alzheimer's disease (AD), Lewybody-related diseases, Pick's disease and Huntington's disease [5-11]. Current understanding suggests that modifications to TDP-43 including hyperphosphorylation and proteolytic cleavage by caspases lead to a toxic gain of function. In particular, truncated TDP-43 redistributes from the nucleus to the cytoplasm [12], and this may promote cellular dysfunction by causing altered trafficking of the protein [13]. Therefore, posttranslational proteolytic processing of TDP-43 by caspases may be a key step in protein misfolding and aggregation of TDP-43 $[13,14]$.

In a recent report, Zhang et al. [12] showed that the ectopic expression of an approximately $25-\mathrm{kDa}$ TDP- 43 fragment corresponding to the $\mathrm{C}$-terminal truncation product of caspase-cleaved TDP-43 leads to the formation of toxic, insoluble and ubiquitin-positive cytoplasmic inclusions within human cell lines. In addition, by generating a conformation-dependent antibody that detects C-terminal fragments, caspase-cleaved TDP-43 was identified in postmortem human brain sections in FTLDU and ALS [12].

We recently developed a site-directed caspase cleavage antibody to TDP-43, termed TDPccp, and identified caspase-cleaved TDP-43 in several tauopathies including AD and Pick's disease [7, 11]. Specifically, caspase-cleaved TDPccp was identified within Hirano bodies in the CA1 region of the hippocampus in $\mathrm{AD}$ and Pick's disease, suggesting this might be a common feature of tauopathies [7, 11]. These findings support the conclusion that the presence of TDP-43 pathology is not solely restricted to TDP43 proteinopathies, but may be more widely distributed in a number of neurodegenerative diseases [13]. The purpose of the present study was to determine a possible role for caspase-cleaved TDP-43 in Parkinson's disease (PD) and dementia with Lewy bodies (DLB), neurodegenerative disorders classified as $\alpha$-synucleinopathies.

PD and DLB are clinically characterized by progressive dementia and/or motor syndromes and exhibit widespread neuronal cell loss. In PD, patients develop extrapyramidal movement disturbances [15], and the diagnosis is based on the presence of 2 of the 3 following clinical features: bradykinesia, resting tremor and rigidity [16]. The pathological hallmark of idiopathic PD is loss of dopaminergic neurons from the substantia nigra (SN) [15]. In DLB, several groups have recognized distinctive clinical features including impairment of attention, problem solving and visuospatial skills associated with loss of neurons from the cortex $[17,18]$. Microscopically, in PD and DLB cell loss is associated with the presence of Lewy body inclusions that are comprised principally of aggregated $\alpha$-synuclein ( $\alpha$-Syn) [19].

In the present study, application of our site-directed caspase cleavage antibody to TDP-43 in postmortem brain sections from PD and DLB revealed the presence of caspase-cleaved TDP-43 in Lewy bodies of all cases examined. Colocalization of TDPccp with an antibody to $\alpha$-Syn was evident within the SN in both $\alpha$-synucleinopathies. Interestingly, the TDPccp antibody detected on average roughly twice the number of Lewy bodies compared to the $\alpha$-Syn antibody. Collectively, these data have identified caspase-cleaved TDP-43 as a primary component of Lewy bodies in PD and DLB, and suggest that the TDPccp antibody is an effective marker for the detection of Lewy bodies.

\section{Materials and Methods}

\section{Antibodies}

The monoclonal mouse $\alpha$-Syn antibody (clone LB509) was purchased from Zymed (San Francisco, Calif., USA). The caspase cleavage product antibody to TDP-43 TDPccp was an in-house antibody that has previously been shown to be a specific marker for caspase-cleaved TDP-43 [7]. This antibody was synthesized based upon a putative caspase cleavage consensus site (DVMD ${ }^{219}$ ) within TDP-43.

\section{Human Subjects}

Autopsy brain tissue from 5 neuropathologically confirmed PD cases and 5 confirmed DLB cases was studied. Human brain tissues used in this study were provided by the Institute for Memory Impairments and Neurological Disorders at the University of California, Irvine.

Immunohistochemistry and Immunofluorescence Microscopy

For immunohistochemical and immunofluorescence studies, free-floating 40 - $\mu \mathrm{m}$-thick serial sections from PD and DLB sub- 
Fig. 1. Caspase-cleaved TDP-43 in PD and DLB. Representative bright-field single labeling utilizing the TDPccp antibody from a PD case $(\mathbf{a}, \mathbf{c})$ and a DLB case $(\mathbf{b}, \mathbf{d})$. Application of the TDPccp antibody revealed labeling in the $\mathrm{SN}$ region within Lewy bodies (arrowheads, $\mathbf{a}$ and $\mathbf{b}$ ) and in the CA1 region of the hippocampus within Hirano bodies (arrows, $\mathbf{c}$ and $\mathbf{d}$ ) as well as Lewy bodies (arrowheads, c and d). All scale bars represent $10 \mu \mathrm{m}$.
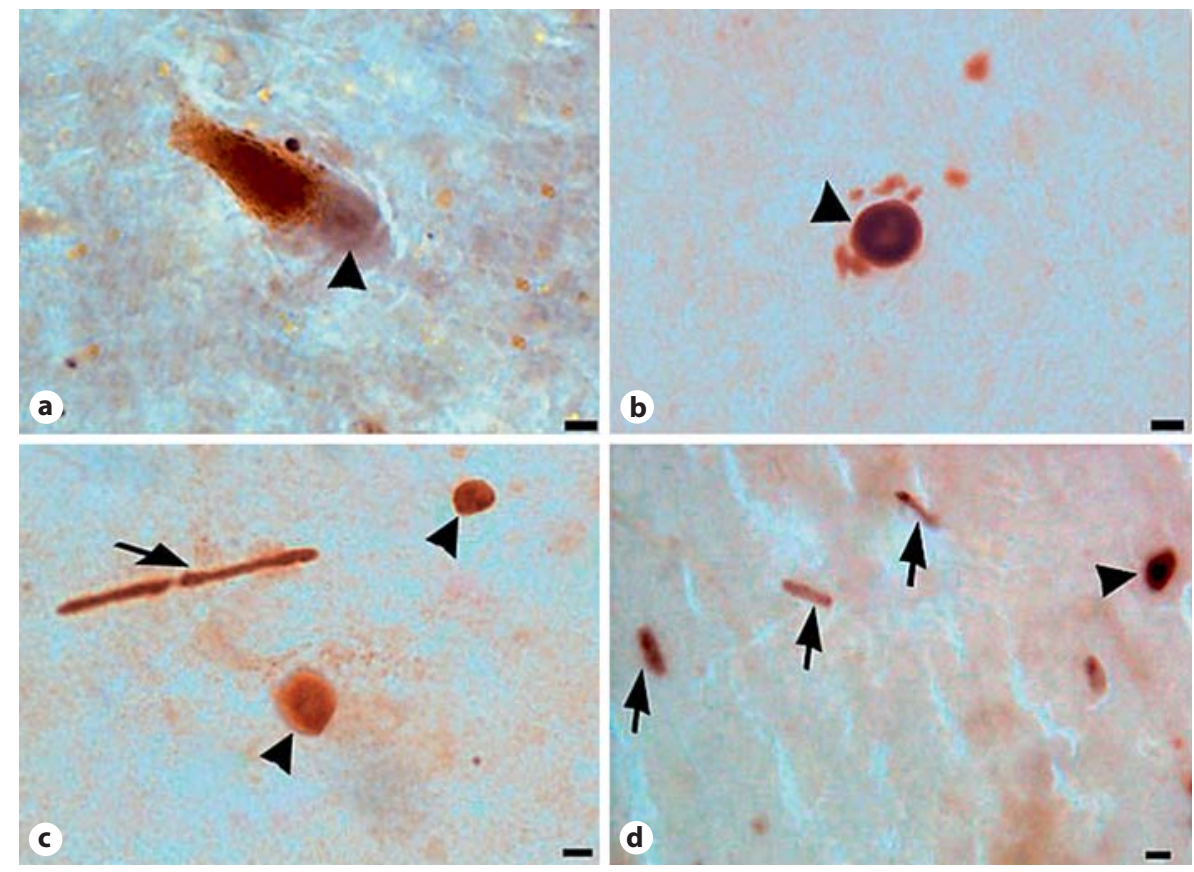

jects were used as previously described [20, 21]. Antibody dilutions were 1:100 for TDPccp and 1:500 for $\alpha$-Syn monoclonal antibody. For single labeling, antigen visualization was accomplished using ABC complex (ABC Elite immunoperoxidase kit, Vector Laboratories), followed by brown/red diaminobenzidine substrate (Vector Laboratories). For immunofluorescence colocalization studies, antigen visualization was accomplished using an Alexa-fluor-488-labeled tyramide (green, Ex/Em = 495/519) for the first label and streptavidin Alexa fluor 555 (red, Ex/Em = 555/565) for the second label, both purchased from Invitrogen (Carlsbad, Calif., USA).

\section{Western Blot Analysis}

Human brain lysates from DLB and age-matched control subjects were processed for Western blot analysis as previously described [21]. Proteins were separated by $12 \%$ sodium dodecyl sulfate polyacrylamide gel electrophoresis and transferred to nitrocellulose. To verify equal loading between samples, transferred slabs were stained in Coomassie blue. Membranes were incubated in TDPccp, and primary antibody was visualized with a goat anti-rabbit horseradisch-peroxidase-linked secondary antibody (1:5,000; Jackson's Laboratory, West Grove, Pa., USA), followed by electrochemiluminescence detection. The protein content from all the samples was analyzed using the bicinchoninic acid assay (Pierce) to ensure equal protein loading. In addition, Western blot analysis was performed utilizing a $\beta$-actin antibody (1:400) as a loading control.

\section{Quantification and Statistical Analysis}

A semiquantitative analysis was performed by first taking $20 \times$ immunofluorescence, overlapping images (labeled with both TDPccp and $\alpha$-Syn) from the SN area in 5 separate PD and 5 DLB cases. Photographs were then analyzed by counting the number of Lewy bodies and calculating the percentage of inclusion bodies labeled for either $\alpha$-Syn or TDPccp antibodies in the entire tissue section for each case. Data were then averaged and \pm SEM was assessed. Additionally, $\alpha$-Syn labeling was utilized as a general marker for Lewy bodies and compared with the number of $\alpha$-Syn-labeled Lewy bodies that colocalized with TDPccp. The statistical difference between percentages of $\alpha$-Syn single-labeled, TDPccp single-labeled and $\alpha$-Syn-TDPccp double-labeled Lewy bodies was analyzed by 1-way ANOVA (general linear model procedure with contrast statement) with statistical significance established at a $\mathrm{p}$ value $<0.05$. Data were normally distributed with no significant difference in variances across the antibody classes. The calculations were performed using a SAS program version 9.1.3 Service Pack 3 for Windows XP-Pro.

\section{Results}

A critical feature of TDP-43 proteinopathies is the presence of cytoplasmic C-terminal fragments of TDP-43 (approx. 25 and $35 \mathrm{kDa}$ ) that have ability to aggregate [22]. One candidate protease that may be involved in the proteolytic processing of TDP-43 is caspase 3. Zhang et al. [23] have shown in vitro that TDP-43 is a substrate for caspase 3 leading to the generation of $25-$ and $35-\mathrm{kDa}$ species. The goal of the present study was to examine whether caspase-cleaved TDP-43 is a relevant finding in the PD and DLB brain. 


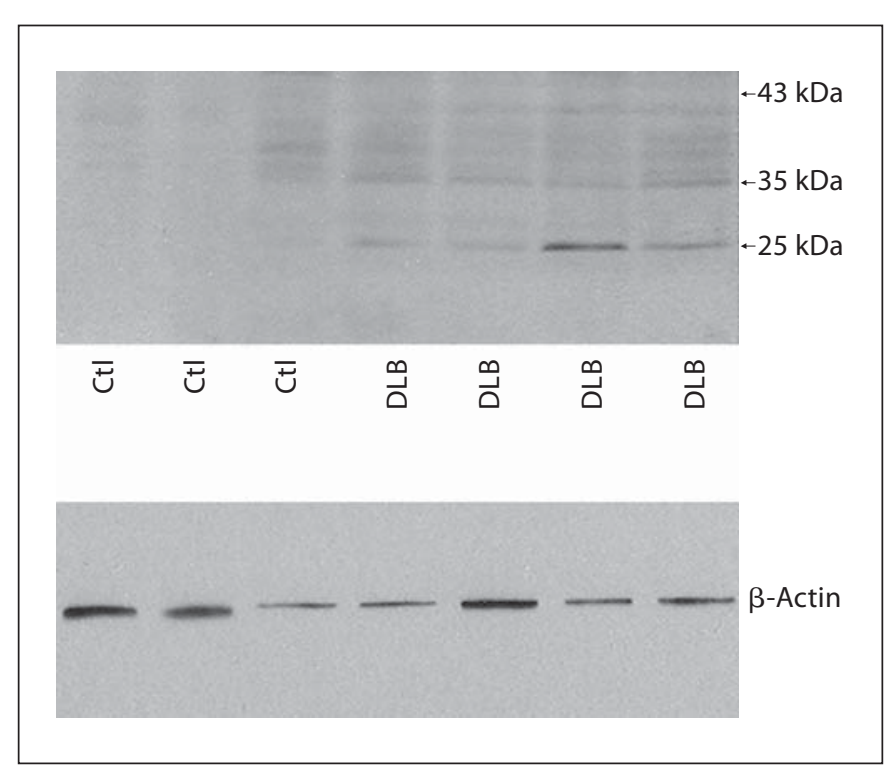

Fig. 2. Western blot analysis utilizing the TDPccp antibody in representative DLB or control $(\mathrm{Ctl})$ cases. Western blot analysis using the TDPccp antibody confirmed the presence of the predicted caspase-cleaved fragment TDP-43 $(25 \mathrm{kDa})$ in all $4 \mathrm{DLB}$ cases. The bottom panel represents the same blot stripped and reprobed with a $\beta$-actin antibody, which served as a loading control. Data are representative of 3 independent experiments.

Five confirmed cases of PD and DLB were examined by immunohistochemistry utilizing our site-directed caspase-cleaved antibody to TDP-43, termed TDPccp. As an initial approach, immunoreactivity to TDPccp was assessed in two different brain regions including the $\mathrm{SN}$ and hippocampus. Application of the TDPccp antibody using bright-field microscopy revealed caspase-cleaved TDP-43 labeling in the SN region within Lewy bodies (arrowheads, fig. 1a, b). In hippocampal sections, caspase-cleaved TDP-43 was found in Lewy bodies and within Hirano bodies in both diseases (arrows, fig. 1c, d). To confirm the immunohistochemical results, Western blot analysis was carried out using the TDPccp antibody. The results confirmed the presence of the predicted caspase-cleaved fragment of TDP-43 $(25 \mathrm{kDa})$ in all $4 \mathrm{DLB}$ cases examined (fig. 2). As shown, the antibody also recognized a fainter band in DLB subjects running at 35 $\mathrm{kDa}$. This may represent an additional caspase-cleaved fragment of TDP-43 as a previous study has shown that caspase cleavage of TDP-43 results in the generation of both $35-$ and $25-\mathrm{kDa}$ fragments [12]. There was no immunoreactivity observed at $43 \mathrm{kDa}$ corresponding to full-length TDP-43 in any of the samples analyzed (con- trol or DLB), suggesting a lack of immunoreactivity of TDPccp to full-length TDP-43.

A common pathological feature of PD and DLB is the presence of $\alpha$-Syn, and numerous studies employ the use of a full-length antibody to $\alpha$-Syn as a general marker for Lewy bodies. We examined the extent of colocalization of TDPccp with a well-characterized full-length antibody to $\alpha$-Syn (LB509, Zymed Labs). Immunofluorescence double-labeling experiments indicated a colocalization of both antibodies within Lewy bodies in PD and DLB (fig. 3). Of interest was the relative uniform distribution of $\alpha$-Syn and TDPccp labeling within Lewy bodies in PD, while in DLB both $\alpha$-Syn and the TDPccp labeling appeared to be more peripherally distributed. These findings confirmed the presence of caspase-cleaved TDP-43 within Lewy bodies in PD and DLB. Although colocalization was evident within the $\mathrm{SN}$ of both diseases, single labeling of Lewy bodies with either antibody was also evident (fig. 4). Moreover, it appeared that more Lewy bodies were singly labeled with TDPccp than with the $\alpha$-Syn antibody (fig. 4). Quantification experiments indicated that the majority of Lewy bodies identified within SN brain sections were labeled with both TDPccp and the $\alpha$-Syn antibody (blue bars, fig. 5). However, quantification of the number of Lewy bodies labeled by $\alpha$-Syn and TDPccp antibodies in PD and DLB cases revealed that the average percentage of caspase-cleaved TDP-43 single-labeled Lewy bodies was approximately twice that of $\alpha$-Syn labeling in both $\alpha$-synucleinopathies (fig. 5a: 13.4 vs. $5.5 \%$ in DLB, fig. 5 b: 34.6 vs. $17.6 \%$ in PD). Statistical differences between the percentages of $\alpha$-Syn single-labeled, TDPccp single-labeled and $\alpha$-Syn-TDPccp double-labeled Lewy bodies was analyzed by 1-way ANOVA (general linear model procedure with contrast statement) with statistical significance established at a $p$ value $<0.05$. Data were normally distributed with no significant difference in variances across the antibody classes. For DLB, the overall $\mathrm{F}_{2,12}$ was 284.07 with $\mathrm{p}<0.05$ and for PD, the overall $\mathrm{F}_{2,12}$ was 26.41 with $\mathrm{p}<0.05$.

\section{Discussion}

TDP-43 has been identified as a major component of ubiquitinated tau-negative inclusions in sporadic FTLDU and ALS [3]. For this common pathology, FTLD-U and ALS are now grouped together as a new entity of neurodegenerative disorders, classified as TDP-43 proteinopathies [24]. Recent advances have suggested that a key event that may promote disease progression in TDP-43 

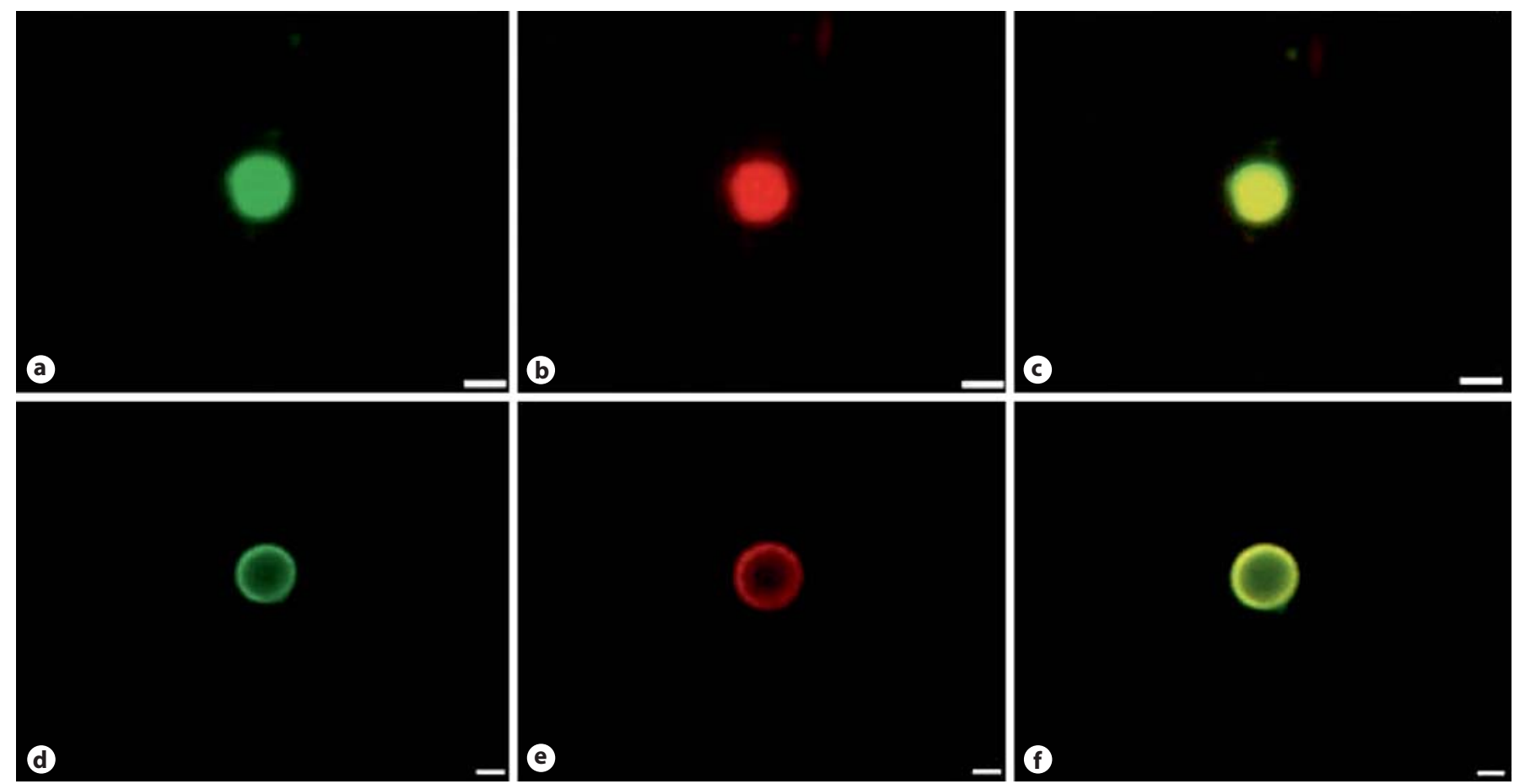

Fig. 3. Caspase-cleaved TDP- 43 colocalizes with $\alpha$-Syn within Lewy bodies in PD and DLB. Full-length $\alpha$-Syn and caspasecleaved TDP-43 antibodies colocalized within Lewy bodies in PD $(\mathbf{a}-\mathbf{c})$ and DLB (d-f). Representative immunofluorescence double labeling employing an $\alpha$-Syn monoclonal antibody (green, a and

proteinopathies is the redistribution of truncated forms of TDP-43 from the nucleus to the cytoplasm [25]. In support of this hypothesis are data demonstrating the presence of smaller (approx. $25 \mathrm{kDa}$ ) phosphorylated fragments of TDP-43 in brain samples from both FTLD-U and ALS [26]. These findings suggest that posttranslational modification of TDP-43 may signal a shift to a toxic gain of function. One candidate protease that may be involved in the proteolytic processing of TDP-43 is caspase 3. Zhang et al. [23] have shown that TDP-43 is a substrate for caspase 3 leading to the generation of 25- and $35-\mathrm{kDa}$ species. Our laboratory has also supported the hypothesis that caspases may play a major role in the processing of TDP-43 in other neurodegenerative disorders: utilizing a site-directed caspase cleavage antibody to a known consensus cleavage site within TDP-43 $\left(D_{V M D}^{219}\right)$, we identified caspase-cleaved TDP-43 in two tauopathies, AD and Pick's disease $[7,11]$. Specifically, caspase-cleaved TDP-43 was identified within Hirano bodies in the CA1 region of the hippocampus in $\mathrm{AD}$ and Pick's disease.

Caspase-Cleaved TDP-43 in PD and DLB d) and TDPccp (red, $\mathbf{b}$ and e) and the overlap image for both markers (yellow, c and f). Note the relative uniform distribution of $\alpha$ Syn and TDPccp labeling within the Lewy body in the PD case (c), while in the DLB case both $\alpha$-Syn and the TDPccp appear to be more peripherally distributed (f). All scale bars represent $10 \mu \mathrm{m}$.

These findings support the conclusion that the presence of caspase-cleaved TDP-43 is not solely restricted to TDP-43 proteinopathies, but may be more widely distributed in a number of neurodegenerative diseases. Thus, to confirm and extend these findings, our goal in the present study was to examine whether caspase-cleaved TDP43 is a relevant finding in PD and DLB, collectively known as $\alpha$-synucleinopathies. In PD and DLB, neurodegeneration is believed to be associated with the presence of intraneuronal inclusions termed Lewy bodies that are composed of $\alpha$-Syn [27]. Numerous studies support the hypothesis that $\alpha$-Syn aggregation is the key step driving pathology, cellular damage and subsequent neuronal dysfunction [28-31]. In the present study, we examined whether an accumulation of caspase-cleaved TDP- 43 is a major finding associated with these $\alpha$-synucleinopathies.

Five confirmed cases of PD and DLB were examined by immunohistochemistry utilizing our site-directed caspase-cleaved antibody to TDP-43, termed TDPccp. As an initial approach, immunoreactivity to TDPccp was assessed in two different brain regions including the SN 

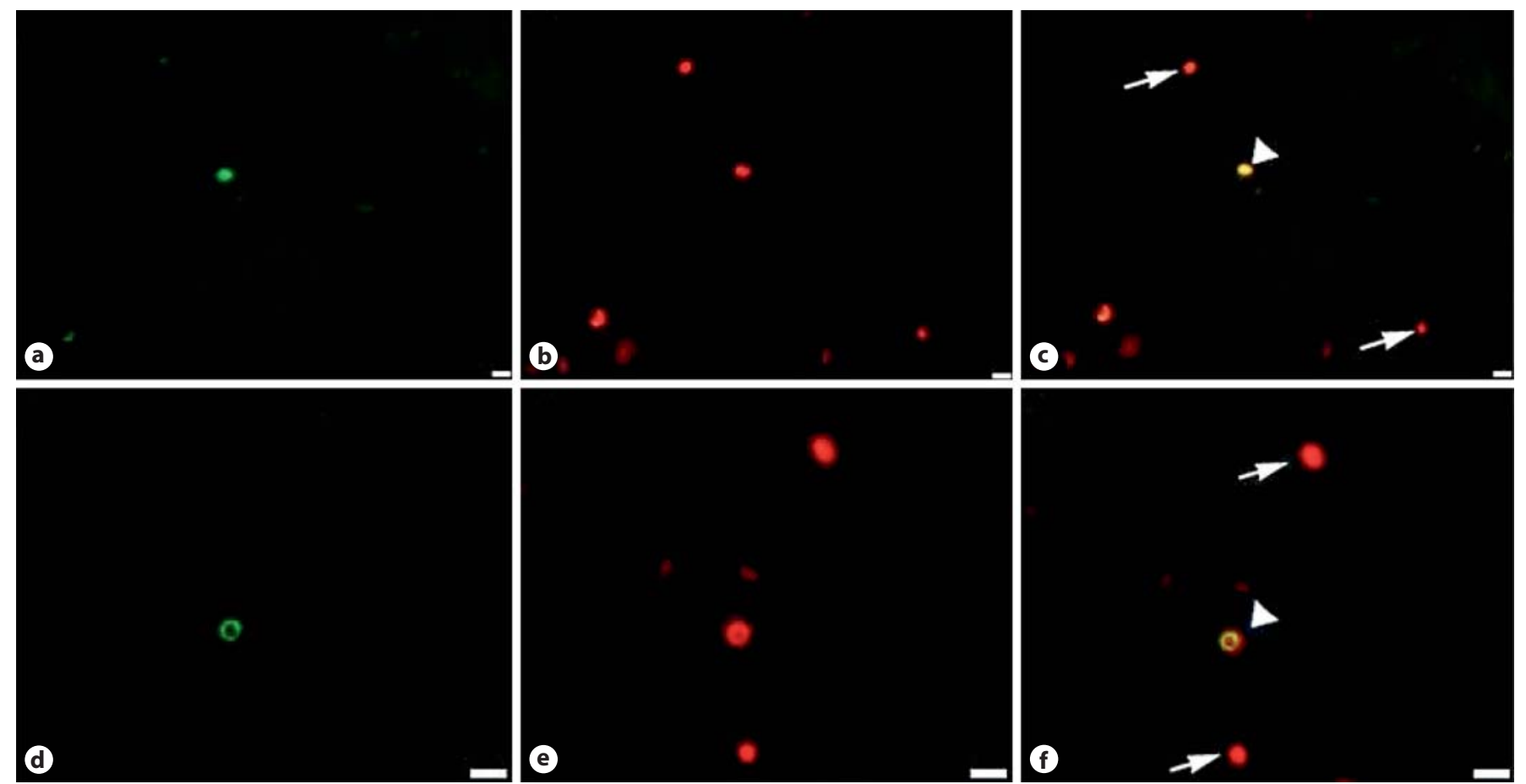

Fig. 4. The TDPccp antibody detects a greater number of Lewy bodies in PD and DLB than the standardized marker $\alpha$-Syn. a-c Representative immunofluorescence double labeling utilizing the $\alpha$-Syn antibody (green), TDPccp (red) and the overlap image (c). $\mathbf{d - f}$ Identical to $\mathbf{a}$-c showing $\alpha$-Syn labeling (green), TDPccp (red) and the overlap image for both markers within a Lewy body (ar-

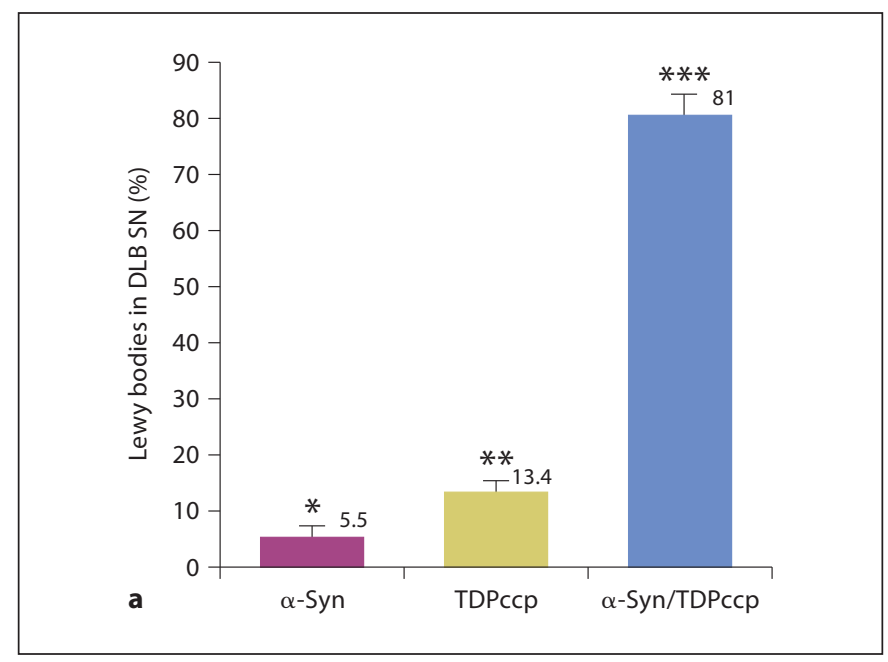

Fig. 5. Caspase-cleaved TDP-43 as a primary marker for Lewy bodies in DLB and PD. Quantification of Lewy bodies labeled by $\alpha$-Syn and TDPccp antibodies. A monoclonal antibody to $\alpha$-Syn was utilized as a marker for Lewy bodies. Data show the average percentage \pm SEM of Lewy bodies labeled with $\alpha$-Syn, TDPccp and with both antibodies in the SN of DLB cases (a) and PD cases (b), with percent given above bars. Note that in both $\alpha$-synucle- rowhead, $\mathbf{f})$. Note the greater number of caspase-cleaved TDP-43labeled Lewy bodies compared to full-length $\alpha$-Syn-labeled Lewy bodies in the $\mathrm{SN}$ in both $\alpha$-synucleinopathies (arrows, c and f). Arrowheads in $\mathbf{c}$ and $\mathbf{f}$ denote the presence of a Lewy body doublelabeled with both antibodies. a-c Scale bars represent $10 \mu \mathrm{m}$. d-f Scale bars represent $20 \mu \mathrm{m}$.

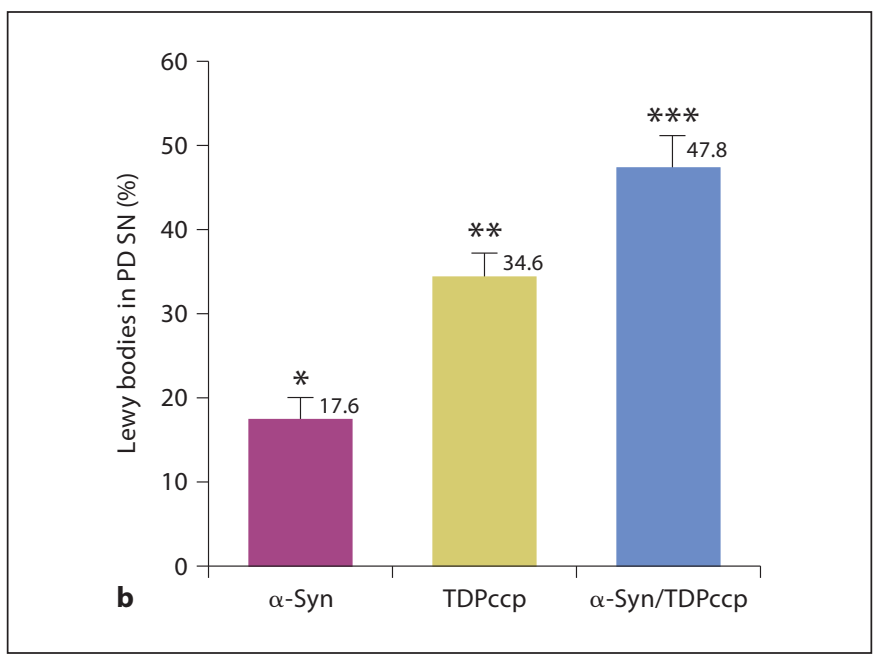

inopathies the percentage of caspase-cleaved TDP-43 single-labeled Lewy bodies is approximately twice that of full-length $\alpha$ Syn labeling. Data represent the average percentage of Lewy bodies $( \pm$ SEM) in whole sections from 5 representative DLB cases $(\mathbf{a}$, overall $\left.\mathrm{F}_{2,12}=284.07, \mathrm{p}<0.05\right)$ and $5 \mathrm{PD}$ cases $\left(\mathbf{b}\right.$, overall $\mathrm{F}_{2,12}=$ 26.41, $\mathrm{p}<0.05)$. 
and hippocampus. Application of the TDPccp antibody using bright-field microscopy revealed the presence of caspase-cleaved TDP-43 within Lewy bodies in the SN of DLB and PD samples. In hippocampal sections, caspasecleaved TDP-43 was found in Lewy bodies and within Hirano bodies in both diseases. Hirano bodies are characterized as rod-shaped, paracrystalline structures in the neurons of the central nervous system and are commonly found in numerous neurodegenerative diseases within the CA1 region of the hippocampus [32]. These results parallel our findings of caspase-cleaved TDP-43 within Hirano bodies in AD and Pick's disease [7, 11] and support a general role for caspase activation and cleavage of TDP-43 within these eosinophilic structures. It is important to note that although structures labeled by our TDPccp antibody morphologically resembled Hirano bodies, no experiments were carried out to confirm this finding using specific markers to Hirano bodies. Therefore, further studies will be necessary to confirm the finding of caspase-cleaved TDP-43 within these structures.

A central feature of TDP- 43 proteinopathies is the presence of cytoplasmic fragments of TDP-43 (approx. 25 and $35 \mathrm{kDa}$ ) that have ability to aggregate [22]. Current understanding suggests that the $25-\mathrm{kDa}$ TDP-43 fragment corresponding to the truncation product of caspase-cleaved TDP-43 leads to a toxic gain of function. For example, caspase cleavage of TDP-43 is a critical step leading to the aggregation and redistribution of TDP-43 from the nucleus to the cytoplasm [12]. In addition, a recent study by Johnson et al. [33] has demonstrated that only aggregating forms of TDP-43 are toxic. Therefore, posttranslational proteolytic processing of TDP-43 by caspases may be a key step in protein misfolding, aggregation and toxicity of TDP-43 in ALS and FTLD-U [13, 14]. Our data showing the presence of caspase-cleaved TDP-43 (approx. 25-kDa fragment) within Lewy bodies extends these findings to $\alpha$-synucleinopathies.

A common pathological feature of PD and DLB is the presence of $\alpha$-Syn, and numerous studies employ the use of a full-length antibody to $\alpha$-Syn as a general marker for Lewy bodies. We examined the extent of colocalization of TDPccp with a well-characterized full-length antibody to $\alpha$-Syn (LB509, Zymed Laboratories). Immunofluorescence double-labeling experiments indicated a colocalization of both antibodies within Lewy bodies in PD and $\mathrm{DLB}$, thereby confirming the presence of caspase-cleaved TDP-43 within Lewy bodies in PD and DLB. Quantification experiments indicated that the majority of Lewy bodies identified within SN brain sections were labeled with both TDPccp and the $\alpha$-Syn antibody. However, a small percentage of Lewy bodies was found which were only labeled with either TDPccp or the $\alpha$-Syn antibody, respectively. Quantification of the number of Lewy bodies single-labeled by TDPccp showed approximately twice the percentage of that of the full-length $\alpha$-Syn antibody in both $\alpha$-synucleinopathies. Statistical differences were detected between the percentages of $\alpha$-Syn single-labeled, TDPccp single-labeled and $\alpha$-Syn-TDPccp double-labeled Lewy bodies by 1-way ANOVA (general linear model procedure with contrast statement) with statistical significance established at a p value $<0.05$. The identification of Lewy bodies that only labeled with TDPccp and are $\alpha$-Syn negative can be interpreted in several ways. One interpretation is the existence of Lewy bodies in the absence of $\alpha$-Syn pathology. Although possible, and in fact previously reported [34], these structures probably represent a minority of the total number of Lewy bodies present following tissue analysis. A more likely interpretation of our data is the presence of Lewy bodies that labeled with TDPccp, but had insufficient levels of $\alpha$-Syn to be detected by the $\alpha$-Syn antibody at the titer employed (1:500). In either case, our data clearly show that the majority of Lewy bodies identified contain both $\alpha$-Syn and caspase-cleaved TDPccp.

In conclusion, results from the present study identify caspase-cleaved TDP-43 as a primary component of Lewy bodies in $\alpha$-synucleinopathies and suggest that the TDPccp antibody is an effective marker for the detection of Lewy bodies. Future studies are necessary, however, to address whether the presence of caspase-cleaved TDP-43 in $\alpha$-synucleinopathies is a cause or effect of the underlying pathological events associated with these neurodegenerative diseases.

\section{Acknowledgements}

This work was funded by NIH/NCRR grant No. P20RR016454 and a grant from the American Health Assistance Foundation to T.T.R. This work was also supported by a gracious donation from the KO AD Foundation (Boise, Idaho, USA) to T.T.R. 


\section{References}

1 Buratti E, Baralle FE: Multiple roles of TDP43 in gene expression, splicing regulation, and human disease. Front Biosci 2008; 13 : 867-878.

-2 Strong MJ, Volkening K, Hammond R, Yang W, Strong W, Leystra-Lantz C, Shoesmith C: TDP43 is a human low molecular weight neurofilament (hNFL) mRNA-binding protein. Mol Cell Neurosci 2007;35:320-327.

-3 Neumann M, Sampathu DM, Kwong LK, Truax AC, Micsenyi MC, Chou TT, Bruce J, Schuck T, Grossman M, Clark CM, McCluskey LF, Miller BL, Masliah E, Mackenzie IR, Feldman H, Feiden W, Kretzschmar HA, Trojanowski JQ, Lee VM: Ubiquitinated TDP-43 in frontotemporal lobar degeneration and amyotrophic lateral sclerosis. Science 2006;314:130-133.

4 Forman MS, Trojanowski JQ, Lee VM: TDP43: a novel neurodegenerative proteinopathy. Curr Opin Neurobiol 2007;17:548-555.

5 Fujishiro H, Uchikado H, Arai T, Hasegawa $\mathrm{M}$, Akiyama H, Yokota O, Tsuchiya K, Togo T, Iseki E, Hirayasu Y: Accumulation of phosphorylated TDP-43 in brains of patients with argyrophilic grain disease. Acta Neuropathol 2009;117:151-158.

-6 Higashi S, Iseki E, Yamamoto R, Minegishi M, Hino H, Fujisawa K, Togo T, Katsuse O, Uchikado H, Furukawa Y, Kosaka K, Arai H: Concurrence of TDP-43, tau and alphasynuclein pathology in brains of Alzheimer's disease and dementia with Lewy bodies. Brain Res 2007;1184:284-294.

7 Rohn TT: Caspase-cleaved TAR DNA-binding protein-43 is a major pathological finding in Alzheimer's disease. Brain Res 2008; 1228:189-198.

-8 Nakashima-Yasuda H, Uryu K, Robinson J, Xie SX, Hurtig H, Duda JE, Arnold SE, Siderow A, Grossman M, Leverenz JB, Woltjer R, Lopez OL, Hamilton R, Tsuang DW, Galasko D, Masliah E, Kaye J, Clark CM, Montine TJ, Lee VM, Trojanowski JQ: Comorbidity of TDP-43 proteinopathy in Lewy body related diseases. Acta Neuropathol 2007;114:221-229.

-9 Schwab C, Arai T, Hasegawa M, Yu S, McGeer PL: Colocalization of transactivationresponsive DNA-binding protein 43 and huntingtin in inclusions of Huntington disease. J Neuropathol Exp Neurol 2008;67: 1159-1165.

10 Uryu K, Nakashima-Yasuda H, Forman MS, Kwong LK, Clark CM, Grossman M, Miller BL, Kretzschmar HA, Lee VM-Y, Trojanowski JQ, Neumann M: Concomitant TARDNA-binding protein 43 pathology is present in Alzheimer disease and corticobasal degeneration but not in other tauopathies. J Neuropathol Exp Neurol 2008;67:555-564.
11 Rohn TT, Kokoulina P: Caspase-cleaved TAR DNA-binding protein-43 in Pick's disease. Int J Physiol Pathophysiol Pharmacol 2009;1:24-31.

12 Zhang Y-J, Xu Y-F, Cook C, Gendron TF, Roettges P, Link CD, Lin W-L, Tong J, Castanedes-Casey M, Ash P, Gass J, Rangachari V, Buratti E, Baralle F, Golde TE, Dickson DW, Petrucelli L: Aberrant cleavage of TDP43 enhances aggregation and cellular toxicity. PNAS 2009;106:7607-7612.

13 Rohn TT: Cytoplasmic inclusions of TDP-43 in neurodegenerative diseases: a potential role for caspases. Histol Histopathol 2009; 24:1081-1086.

14 Kwong LK, Uryu K, Trojanowski JQ, Lee VM: TDP-43 proteinopathies: neurodegenerative protein misfolding diseases without amyloidosis. Neurosignals 2008;16:41-51.

15 Dawson TM, Dawson VL: Molecular pathways of neurodegeneration in Parkinson's disease. Science 2003;302:819-822.

16 McKeith IG, Galasko D, Kosaka K, Perry EK, Dickson DW, Hansen LA, Salmon DP, Lowe J, Mirra SS, Byrne EJ, Lennox G, Quinn NP, Edwardson JA, Ince PG, Bergeron C, Burns A, Miller BL, Lovestone S, Collerton D, Jansen EN, Ballard C, de Vos RA, Wilcock GK, Jellinger KA, Perry RH: Consensus guidelines for the clinical and pathologic diagnosis of dementia with Lewy bodies (DLB): report of the consortium on DLB international workshop. Neurology 1996;47:1113-1124.

-17 Crystal HA, Dickson DW, Lizardi JE, Davies P, Wolfson LI: Antemortem diagnosis of diffuse Lewy body disease. Neurology 1990;40: 1523-1528.

18 Hansen L, Salmon D, Galasko D, Masliah E, Katzman R, De Teresa R, Thal L, Pay MM, Hofstetter R, Klauber M, Rice V, Butters N, Alford M: The Lewy body variant of Alzheimer's disease: a clinical and pathologic entity. Neurology 1990;40:1-8.

19 Dickson DW: Alpha-synuclein and the Lewy body disorders. Curr Opin Neurol 2001;14: 423-432.

-20 Rohn TT, Rissman RA, Davis MC, Kim Y-E, Cotman C, Head E: Caspase-9 activation and caspase cleavage of tau in the Alzheimer's disease brain. Neurobiol Dis 2002;11:341354.

21 Mouser PE, Head E, Ha K-H, Rohn TT: Caspase-mediated cleavage of glial fibrillary acidic protein within degenerating astrocytes of the Alzheimer's disease brain. Am J Pathol 2006;168:936-946.

22 Winton MJ, Igaz LM, Wong MM, Kwong LK, John Q, Trojanowski JQ, Virginia M-Y, Lee VM-Y: Disturbance of nuclear and cytoplasmic TAR DNA-binding protein (TDP-43) induces disease-like redistribution, sequestration, and aggregate formation. J Biol Chem 2008;283:13302-13309.
23 Zhang Y-J, Xu Y-F, Dickey CA, Buratti E, Baralle F, Rachel Bailey R, Stuart PickeringBrown S, Dickson D, Petrucelli L: Progranulin mediates caspase-dependent cleavage of TAR DNA binding protein-43. J Neurosci 2007;27:10530-10534.

24 Liscic RM, Grinberg LT, Zidar J, Gitcho MA, Cairns NJ: ALS and FTLD: two faces of TDP43 proteinopathy. Eur J Neurol 2008;15:772780 .

25 Banks GT, Kuta A, Isaacs AM, Fisher EMC: TDP-43 is a culprit in human neurodegeneration, and not just an innocent bystander. Mamm Genome 2008;19:299-305.

26 Kwong LK, Neumann M, Sampathu DM, Lee VM, Trojanowski JQ: TDP-43 proteinopathy: the neuropathology underlying major forms of sporadic and familial frontotemporal lobar degeneration and motor neuron disease. Acta Neuropathol 2007;114:63-70.

27 Spillantini MG, Schmidt ML, Lee VM, Trojanowski JQ, Jakes R, Goedert M: Alphasynuclein in Lewy bodies. Nature 1997;388: 839-840.

28 Dufty BM, Warner LR, Hou ST, Jiang SX, Gomez-Isia T, Leenhouts KM, Oxford JT, Feany MB, Masliah E, Rohn TT: Calpaincleavage of $\alpha$-synuclein: connecting proteolytic processing of disease-linked aggregation. Am J Pathol 2007;170:1725-1738.

29 Lundvig D, Lindersson E, Jensen PH: Pathogenic effects of alpha synuclein aggregation. Brain Res Mol Brain Res 2005;134:3-17.

30 Cookson MR: The biochemistry of Parkinson's disease. Annu Rev Biochem 2005;74: 29-52.

31 Lee MK, Stirling W, Xu Y, Xu X, Qui D, Mandir AS, Dawson TM, Copeland NG, Jenkins NA, Price DL: Human alpha-synuclein-harboring familial Parkinson's disease-linked Ala-533 Thr mutation causes neurodegenerative disease with alpha-synuclein aggregation in transgenic mice. Proc Natl Acad Sci USA 2002;99:8968-8973.

- 32 Hirano A: Hirano bodies and related neuronal inclusions. Neuropathol Appl Neurobiol 1994;20:3-11.

33 Johnson BS, McCaffery JM, Lindquist S, Gitler AD: A yeast TDP-43 proteinopathy model: exploring the molecular determinants of TDP-43 aggregation and cellular toxicity. PNAS 2008;105:6439-6444.

34 Van Duinen SG, Lammers GJ, Maat-Scieman ML, Roos RA: Numerous and widespread alpha-synuclein-negative Lewy bodies in an asymptomatic patient. Acta Neuropathol 1999;97:533-539. 
